\title{
RECOGNITION OF SYLLABLE-CONTRACTED WORDS IN SPONTANEOUS SPEECH USING WORD EXPANSION AND DURATION INFORMATION
}

\author{
Wei-Bin Liang, Chung-Hsien Wu, Yu-Kai Kang \\ Department of Computer Science and Information Engineering, \\ National Cheng Kung University, Tainan \\ p7892115@mail.ncku.edu.tw, chwu@csie.ncku.edu.tw,p76954571@mail.ncku.edu.tw
}

\begin{abstract}
This paper presents a graphical model-based approach to syllable-contracted (SC) word recognition of spontaneous Mandarin speech. Phone deletion and pronunciation reduction are two major effects for the syllable-contracted words in spontaneous speech. In this study, the syllablecontracted (SC) words selected from a collected corpus are used for pronunciation lexicon expansion to deal with the phone deletion problem. The duration information of SC words is then incorporated to cover the effect of pronunciation reduction. The graphical model is employed to rescore all possible word sequences, including the expanded SC words, to obtain the final word sequence. In the experimental results, the Mandarin Conversional Dialogue Corpus (MCDC) was used to evaluate the proposed method. Compared with the previous work, a satisfactory improvement on the performance of the proposed approach can be achieved.
\end{abstract}

Index Terms - syllable contraction, duration information, graphical model, spontaneous speech

\section{INTRODUCTION}

Speech is one of the most fundamental and natural communication means of human beings. With the exponential growth in available computing power and significant progress in speech technology, high performance automatic speech recognition (ASR) has been achieved for read speech. However, there is still a great gap between read and spontaneous speech recognition. Compared to the read speech, the speaking mode of spontaneous speech is more complex. An immediate observation is that spontaneous speech is pronounced faster than read speech. Bernstein et al. [1] showed that the pronunciations in spontaneous speech are different from the fast read speech. They also indicated that most speakers tend to delete phones rather than reduce durations during spontaneous speech. Phone deletion and pronunciation reduction, which generally result in syllable contraction, are the two major effects in spontaneous speech and syllable contraction is one of the most difficult problems in spontaneous speech recognition. By investigating the characteristics of syllable contraction, the syllable-contracted words sometimes sound like not just a monosyllabic word. The property of syllable-contracted words is highly dependent on the number of contracted syllables and their syllable structures.

Prior research related to syllable contraction issue could be divided into several categories based on the knowledge sources employed: phonetics, pronunciation lexicon, and acoustic model expansion. In the first category, Tseng introduced that syllable contraction is a continuous process with a predictable target syllable by means of quantitative analyses and acoustic measurement [2][3]. Tseng also proposed that morphological distribution influences syllable in a contraction. Jurafsky et al. [4] presented that most frequent words with pronunciation reduction are also the most frequent words in the corpus. These words are mostly function words and meaningless. In the secondary category, syllable contraction issues were transformed into a pronunciation variation issue. Tsai et al. [5] made a survey that the pronunciation variation information can be incorporated into the pronunciation lexicon either explicitly or implicitly. They also proposed a three-stage framework including generation, ranking, and pruning, for pronunciation modeling to expand the pronunciation lexicon. Liu et al. [6] adapted the decision tree based pronunciation modeling from English to Mandarin to predict alternate pronunciation. In acoustic model expansion, Sun et al. [7] proposed syllable pair acoustic model (SPAM) to model the contracted syllables. Lo et al. [8] adopted kernel Principle Component Analysis (PCA) for model splitting to include more acoustic level units. Besides, some approaches tried to improve the performance of spontaneous speech recognition. Soong et al. [9] proposed the general word posterior probability (GWPP) to measure the reliability of the words in a word lattice. Zhou et al. [10] presented the language model based approach to correcting the error detected by GWPP.

This paper addresses the problem of word recognition errors due to syllable contraction in spontaneous speech recognition. For pronunciation lexicon expansion, this study considers the recognition outputs from the ASR including either normally pronounced syllables or the contracted syllables in the SC words. The highly frequent 
misrecognized SC words are used for the modeling of lexicon expansion. Besides, the duration information for each SC words is thus incorporated for duration modeling. The experimental results show that a reasonable improvement on the recognition performance of SC words can be obtained.

The rest of this paper is organized as follows. Section 2 introduces the framework of detection and correction of syllable contracted words, which takes the duration information of speech models and expand the SC-word candidates into account for speech recognition. Section 3 presents the experiments to evaluate the proposed approach. Finally, the concluding remarks are made in Section 4.

\section{Modeling and Recognition of SC Words}

The conventional approach to speech recognition is generally based on the maximum a posteriori (MAP) method as follows.

$$
\mathbf{W}^{*}=\underset{\mathbf{W}}{\arg \max } P(\mathbf{W} \mid \mathbf{X})=\underset{\mathbf{W}}{\arg \max } P(\mathbf{X} \mid \mathbf{W}) P(\mathbf{W})
$$

where the recognized word sequence $\mathbf{W}^{*}=w_{1}, \ldots, w_{N}$ is composed of $N$ words corresponding to a given sequence of acoustic feature vectors $\mathbf{X}=\mathbf{x}_{1}, \ldots, \mathbf{x}_{N}$. The recognized word sequence $\mathbf{W}^{*}$ is obtained by maximizing the posterior probability $P(\mathbf{W} \mid \mathbf{X})$, which can be decomposed into two components with $P(\mathbf{X} \mid \mathbf{W})$ representing the acoustic models and $P(\mathbf{W})$ being the language model. Considering the effect of syllable contraction in spontaneous speech, phone deletion and pronunciation reduction of speech units play important roles in discriminating $\mathrm{SC}$ word from the normal word. Accordingly, the SC words misrecognized into other words are likely to have the effect of phone deletion and should be treated as a new word and included in the pronunciation lexicon. In addition, the duration information of an SC word should be incorporated into the recognition procedure to deal with the problem of pronunciation reduction. Therefore, $\mathrm{Eq}(1)$ can be re-written as

$$
\mathbf{W}^{*}=\underset{\mathbf{W}}{\arg \max } P(\mathbf{X} \mid \mathbf{S C}, \mathbf{W}) P(\mathbf{W})
$$

For the recognition of spontaneous speech with SC words, a graphical model instead of joint probability is employed to characterize the conditional probability $P(\mathbf{X} \mid \mathbf{S C}, \mathbf{W})$. The graphical model which integrates the probability theory and graph theory [11] can construct economically the representation of joint probability distribution by a parentchild relationship. Figure 1 presents the realization of Eq. (2) and the difference to Hidden Markov Model (HMM).

Considering word level modeling, $\mathrm{Eq}(2)$ can be rewritten as

$$
\mathbf{W}^{*}=\underset{\mathbf{W}, S C}{\arg \max } \prod_{n=1}^{N} P\left(\mathbf{x}_{n} \mid w_{n}\right) P\left(\mathbf{x}_{n} \mid S C_{n}\right) P\left(S C_{n} \mid w_{n}\right) P\left(w_{n} \mid w_{n-1}\right)
$$

where $P\left(\mathbf{x}_{n} \mid w_{n}\right)$ represents the recognition score of word $w_{n}$ with respect to speech segment $\mathbf{x}_{n}$. For simplicity, in the recognition process, only the best $\mathrm{SC}$ word $S C_{n}^{k}$ out of all the SC words $\mathbf{S C}=\left\{S C_{n}^{1}, \ldots, S C_{n}^{K}\right\}$ is obtained.

$$
\mathbf{W}^{*}=\underset{\mathbf{W}, \mathbf{D}, \mathbf{k}}{\arg \max } \prod_{n=1}^{N} P\left(\mathbf{x}_{n} \mid w_{n}\right) P\left(\mathbf{x}_{n} \mid S C_{n}^{k}\right) P\left(S C_{n}^{k} \mid w_{n}\right) P\left(w_{n} \mid w_{n-1}\right)
$$

where $P\left(\mathbf{x}_{n} \mid S C_{n}^{k}\right)$ represents the probability of the speech segment $\mathbf{x}_{n}$ assigned to the $k$-th $\mathrm{SC}$ word $S C_{n}^{k}$. This probability can be characterized by an $\mathrm{SC}$-word duration model (SCDM). $P\left(S C_{n}^{k} \mid w_{n}\right)$ represents the probability of the $\mathrm{SC}$ word expanded from the word $w_{n}$ and can be characterized by an SC-word expansion model (SCEM). Figure 2 illustrates the block diagram for the recognition of spontaneous speech with SC words. First, the speech recognizer transcribes the speech signal into an $N$-best word list. All probable words in the list are used to construct a word graph. Then, if the word $w_{n}$ corresponds to SC word candidate $\overline{C W}_{m}$, SCEM is employed to expand the arcs to either a normally pronounced word $S C_{n}^{0}$ or the potential SC words $S C_{n}^{k}$ based on the SC-word candidates $\overline{C W}_{m}$. Finally, the SCDM and SCEM are employed to search the optimal word sequence $\mathbf{W}^{*}$.

\subsection{SC WORD EXPANSION}

For SC-word recognition, SCEM is employed to select the $\mathrm{SC}$-word candidates for pronunciation lexicon expansion. Figure 3 illustrates the training procedure of SCEM. In the first step, $K$ most frequent SC-words set $\mathbf{S C}=\left\{S C^{1}, \ldots S C^{K}\right\}$ are selected from the Mandarin Conversational Dialogue Corpus (MCDC). Next, speech segments $\mathbf{x}_{i}^{k}$ corresponding to the $k$-th SC-word $S C^{k}$ are extracted by aligning between the transcriptions (Tra) and the speech utterances (Utt) in MCDC. Then, in order to cover the recognition errors, the $i$-th speech segment in MCDC aligned to $S C^{k}$ is re-recognized to obtain Top-J recognized word candidates as $\mathbf{C W}_{i}^{k}=\left\{C W_{i, 1}^{k}, \ldots, C W_{i, J}^{k}\right\}$. For all the speech segments in MCDC aligned to $S C^{k}$, we have the word candidate set $\mathbf{C W}^{k}=\left\{\mathbf{C} \mathbf{W}_{1}^{k}, \ldots, \mathbf{C W}_{I_{k}}^{k}\right\}$, where $I_{k}$ denotes the number of speech segment set $\mathbf{x}^{k}$ corresponding to the $k$-th SC-word $S C^{k}$ in MCDC. Due to the problem of data sparseness for $\mathbf{C W}^{k}$, a likelihood measure is proposed in the following to approximate the conditional probability of the candidate SC words given the $k$-th SC-word $S C^{k}$ 


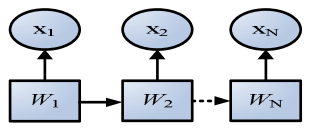

(a) HMM

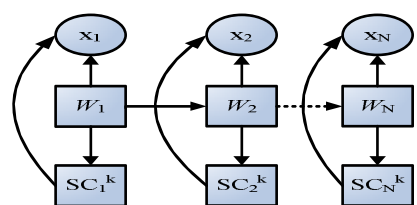

(b) Graphical Model
Figure 1: Speech Recognition via (a) HMM and (b) Graphical Model

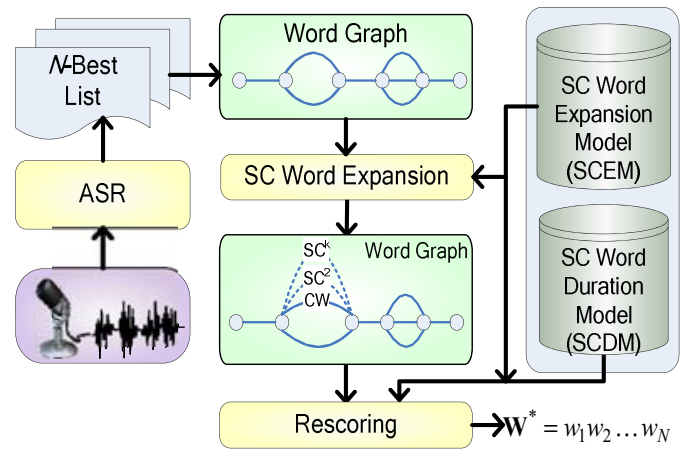

Figure 2: Framework of the proposed approach to spontaneous speech recognition with $\mathrm{SC}$ words

$$
P\left(\overline{C W}_{n}^{k} \mid S C^{k}\right) \equiv \frac{\sum_{i=1}^{R^{k}}\left(1 / \operatorname{Rank}\left(C W_{i, j}^{k}\right)\right)}{\sum_{j=1}^{J} \sum_{i=1}^{I_{k}}\left(1 / \operatorname{Rank}\left(C W_{i, j}^{k}\right)\right)}
$$

where $\operatorname{Rank}\left(C W_{i, j}^{k}\right)$ represents the rank of $C W_{i, j}^{k}$ in the recognition results for the $\mathrm{k}$-th $\mathrm{SC}$-word $S C^{k}$ and $R^{k}$ denotes the number of occurrences of $C W_{i, j}^{k}$ in $\mathbf{C W}^{k}$. $P\left(\overline{C W}_{m}^{k} \mid S C_{n}^{k}\right)$ indicates the probability of the $m$-th SC-word candidate $\overline{C W}_{m}^{k}$ corresponding to the SC word $S C^{k}$. In this study, only $M \mathrm{SC}$-word candidates are selected into the pronunciation lexicon. Then, the $\mathrm{SC}$-word expansion probability $P\left(S C_{n}^{k} \mid w_{n}\right)$ in $\mathrm{Eq}(4)$ is approximated by $P\left(S C_{n}^{k} \mid \overline{C W}_{m}\right)$ as follows.

$P\left(S C_{n}^{k} \mid w_{n}\right) \approx P\left(S C_{n}^{k} \mid \overline{C W}_{m}\right)=P\left(\overline{C W}_{m} \mid S C^{k}\right) \frac{P\left(S C^{k}\right)}{P\left(\overline{C W}_{m}\right)}$

where $\overline{C W}_{m}$ is the recognized SC-word candidate with respect to $w_{n}, P\left(S C^{k}\right)$ is the prior probability of the $k$-th SC-word and $P\left(\overline{C W}_{m}\right)$ is the prior probability of the $m$-th SC word candidate $\overline{C W}_{m}$. Besides, $S C^{0}$ denotes that $\overline{C W}_{m}$ is a normally pronounced word. Finally, all SC-word candidates are collected to be included into the lexicon. For word graph scoring, the lexicon is used to check if the word arcs are SCword candidates.

\subsection{Duration Modeling OF SC WORDS}

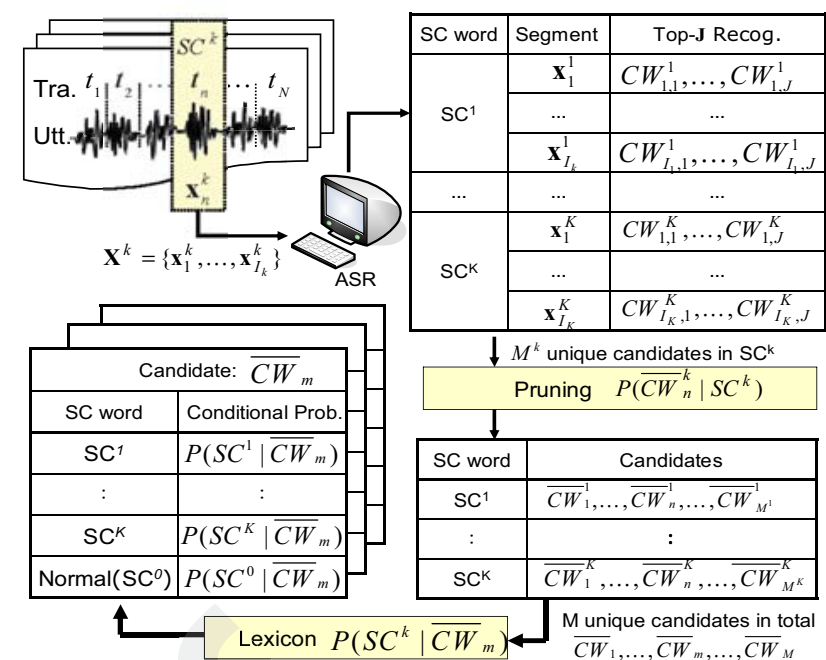

Figure 3: Construction of the pronunciation expansion lexicon in SCEM

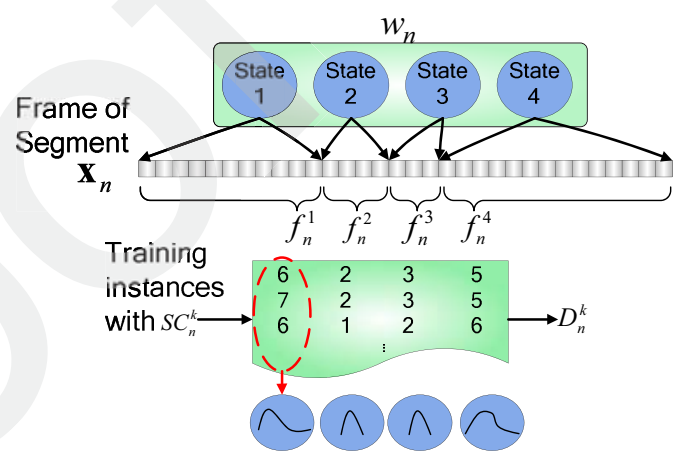

Figure 4: Illustration of duration modeling

SCDM is proposed to discriminate the duration of the normally pronounced words from the SC words. Figure 4 shows the flowchart for SCDM training. Assume that a speech segment $\mathbf{x}_{n}$ is assigned to word $w_{n}$ which is either an SC word or a normal one. $f_{n}^{l}$ denotes the number of frames which are aligned to the $l$-th state of $w_{n}$. The Gamma distribution is adopted to model the frame distribution in each state. The duration information is then defined as:

$$
P\left(\mathbf{x}_{n} \mid S C_{n}^{k}\right)=\left(\prod_{l=1}^{L} \operatorname{Gamma}\left(f_{n}^{l} ; \mu_{n}^{k, l}, \Sigma_{n}^{k, l}\right)\right)^{1 / L}
$$

(7)

where $L$ denotes the number of frames in the state.

By means of these two additional models SCDM and SCEM, the problems of phone deletion and pronunciation reduction can be eliminated.

\section{EXPERIMENT AND RESULTS}

For evaluation, the Mandarin conversational dialogue corpus (MCDC) [12], a popular conversational speech 
Table 1: Recognition results of syllable level and word level (a) SYLLABLE LEVEL

\begin{tabular}{|c|c|c|c|c|c|c|c|c|}
\hline case & Corr & Acc & Hit & Del & Sub & Ins & $\begin{array}{c}\text { Max } \\
\text { acc }\end{array}$ & $\begin{array}{c}\text { Min } \\
\text { acc }\end{array}$ \\
\hline Baseline & 46.28 & 38.55 & 46.28 & 11.04 & 42.67 & 7.73 & 40.15 & 37.37 \\
\hline SCDM & 48.46 & 40.31 & 48.46 & 10.30 & 41.25 & 8.15 & 41.92 & 38.95 \\
\hline SCEM & 49.15 & 40.55 & 49.15 & 9.77 & 41.08 & 8.61 & 42.56 & 38.39 \\
\hline Both & 50.46 & 41.46 & 50.46 & 9.29 & 40.24 & 9.00 & 43.48 & 39.15 \\
\hline
\end{tabular}

(b) WORD LEVEL

\begin{tabular}{|c|c|c|c|c|c|c|c|c|}
\hline case & Corr & Acc & Hit & Del & Sub & Ins & $\begin{array}{c}\text { Max } \\
\text { acc }\end{array}$ & $\begin{array}{c}\text { Min } \\
\text { acc }\end{array}$ \\
\hline Baseline & 33.35 & 26.31 & 33.35 & 10.34 & 56.30 & 7.04 & 28.00 & 24.99 \\
\hline SCDM & 36.15 & 28.66 & 36.15 & 9.62 & 54.23 & 7.49 & 30.34 & 26.90 \\
\hline SCEM & 36.66 & 28.66 & 36.66 & 9.15 & 54.19 & 8.00 & 30.66 & 26.14 \\
\hline Both & 38.42 & 29.98 & 38.42 & 8.71 & 52.87 & 8.43 & 32.05 & 27.27 \\
\hline
\end{tabular}

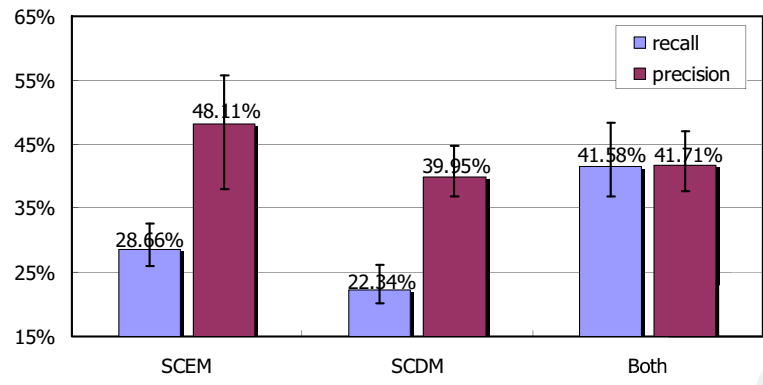

Figure 5: Precision and Recall rates

corpus recorded by Academia Sinica is adopted. MCDC consists of 30 dialogue sets in which eight sets was used to evaluate the proposed method under the 10-fold approach. Of 81,587 syllables in MCDC, 25,111 syllables were annotated with syllable contraction. In this work, an HTKbased speech recognition system (baseline system) was constructed which contains 153 sub-syllable, 37 particle and 13 filler models for Mandarin speech. In addition, 47 most frequent SC words $(K=47)$ and 25 candidates $(M=25)$ were also included in the pronunciation lexicon.

The evaluation results on four approaches including baseline (the HTK-based system), baseline with the proposed SCDM, baseline with SCEM, and baseline with both of SCDM and SCEM are shown in Table 1. The definitions of performance analysis can be referenced to the HTK book. We can observe that the deletion and substitution errors can be improved by including the $\mathrm{SC}$-word models into the pronunciation lexicon. Moreover, the SCDM is able to discriminate the normal syllables from the contracted syllables using the duration models.

An attractive result is that the approach combining SCDM and SCEM obtains the highest performance. The results imply that SCEM with expanded SC words is useful to cover the phone deletion problem and SCDM is beneficial to eliminate the effect of pronunciation reduction. Another interesting result is the performance of precision and recall rates shown in Figure 5. The combined approach improves the recall rate significantly. The result indicates more correct words can be retrieved.

\section{CONCLUSION}

This study presents an approach to the recognition of syllable-contracted words based on SC word expansion and duration information. The $\mathrm{SC}$ words can be first selected from the MCDC corpus. The SC word candidates can be expanded and included in the pronunciation lexicon to deal with the phone deletion problem. The duration information is incorporated to eliminate the pronunciation reduction effect. Finally, a graphical-model-based approach is adopted to rescore all probable word sequences to obtain the best word sequence. The MCDC was used to evaluate the proposed approach. For comparison, the approach combining SCDM and SCEM can achieve a better improvement compared to the previous work.

\section{REFERENCE}

[1] J. Berstein, G. Baldwin, W. Cohen, H. Murveit, and M Weintraub, "Phonological studies for speech recognition," DARPA Speech Recognition Workshop, pp. 41-48, 1992

[2] S.-C Tseng , "Features of Contracted Syllables of Spontaneous Mandarin," in the Proc. of EUROSPEECH2003, pp. $77-80,2003$

[3] S.-C Tseng, "Contracted Syllables in Mandarin: Evidence from Spontaneous Conversation," Journal of Language and Linguistics, pp. 153-180, 2005

[4] D. Jurafsky, A. Bell, M.Gregory, and W.D. Raymond, "The Effect of Language Model Probability on Pronunciation Reduction," in the Proc. of IEEE ICASSP, pp. 801-804, 2001

[5] M.-Y. Tsai, F.-C. Chou, and L.-S. Lee, "Pronunciation Modeling With Reduced Confusion for Mandarin Chinese Using Three-Stage Framework," IEEE Transaction on Audio, Speech and Language Processing, pp. 661-675, 2007

[6] Yi Liu, and Pascale Fung, "Pronunciation Modeling for Spontaneous Mandarin Speech Recognition," International Journal of Speech Technology, pp. 155-172, 2004

[7] L.-Y. Sun, and Y.-R. Wang, "An Analysis Modeling of Syllable Contraction in Spontaneous Mandarin Speech Recognition," Master Thesis, Dept. of Communication Engineering, NCTU, Taiwan, 2004

[8] Y.-S. Lo, and Y.-R. Wang, "An Implementation of Spontaneous Mandarin Speech Recognition Baseline System," Master Thesis, Dept. of Communication Engineering, NCTU, Taiwan, 2005

[9] F.K. Soong, W.-K. Lo, and S. Nakamura, "Generalized Word Posterior Probability For Measuring Reliability Of Recognized Word," in the Proc. of SWIM2004, 2004

[10] Z.-Y. Zhou, Helen Meng, and W.-K. Lo, "A Multi-Pass Error Detection and Correction Framework for Mandarin LVCSR," in the Proc. of IEEE ICSLP, 2006

[11] Michael I. Jordan, "An Introduction to Probabilistic Graphical Models," MIT Press, 1999

[12] S.-C Tseng, and Y.-F. Liu, "Annotation of Mandarin Conversational Dialogue Corpus," CKIP Technical Report, No. 02-01, Academia Sinica, 2002 